\title{
An Immunohistochemical Study of the Distribution of Intermediate Filaments in the Ovary of the Emu (Dromaius novaehollandiae)
}

\section{M.-C. Madekurozwa1*}

\section{Summary}

The immunohistochemical localization of the intermediate filaments desmin, vimentin and smooth muscle actin (SMA) in the ovary of the emu was described in the present study. The cortical region of the ovary contained developing and atretic primordial, pre-vitellogenic and vitellogenic follicles. Vimentin immunostaining was demonstrated in the granulosa cell layer of primordial, previtellogenic and vitellogenic developing and atretic follicles. An interesting finding of the present study was the localization of SMA in fibroblasts located in the theca externa of late vitellogenic follicles. The presence of SMA in these fibroblasts suggests that they possess characteristics of smooth muscle cells.

\section{Introduction}

It is known that intermediate filaments are involved in cellular differentiation and support (Goldman et al., 1996; Galou et al., 1997). Several studies on both avian and mammalian ovaries have demonstrated the presence of intermediate filaments in the surface epithelium, follicular cells and smooth muscle cells (Van Nassauw et al., 1989, 1991, 1992; Van den Hurk et al., 1995; Khan-Dawood et al., 1996; Marettova and Maretta, 2002; Madekurozwa and Kimaro, 2006a). A recent study on the ostrich ovary has shown that the distribution and immunostaining of the intermediate filaments, desmin, vimentin and smooth muscle actin (SMA), changes during follicular development and atresia (Madekurozwa and Kimaro, 2006a). The results of the study suggested that as follicles undergo atresia the intermediate filaments desmin and vimentin are dismantled.

Research conducted on the ovaries of the Japanese quail (Van Nassauw and Callebaut, 1991) and the ostrich (Madekurozwa and Kimaro, 2006a) has demonstrated the presence of SMA in thecal fibroblasts. These findings lend credence to the assertion that thecal fibroblasts possess characteristics of smooth muscle cells. Although certain similarities were observed in the distribution of intermediate filaments in the Japanese quail (Van Nassauw and Callebaut, 1991) and the ostrich (Madekurozwa and Kimaro, 2006a), several differences were noted. This indicates that the distribution of intermediate filaments in the avian ovary is species dependent. Thus, the present study was undertaken to describe the distribution of the intermediate filaments desmin, vimentin and SMA in the ovary of an economically important ratite, the emu. The results of this study will add further information on changes in the localization of intermediate filaments 
during folliculogenesis and regression, which might lead to a better understanding of the role of intermediate filaments in the ovaries of birds.

\section{Materials and Methods}

A total of 11 female emus aged 18 months and weighing $35-40 \mathrm{~kg}$ were used in the present study. The birds originated from a commercial farm in the north-west Province of South Africa. All the birds had active ovaries, which contained approximately 30 predominantly yellow-yolk follicles, with the diameters of the largest follicles ranging from 2 to $3 \mathrm{~cm}$. The birds were sampled in March. The emu being a short-day breeder typically begins to lay in April in the southern hemisphere (Minnaar and Minnaar, 1997).

The birds were slaughtered at a commercial abattoir, employing a standard slaughter protocol. Ovarian tissue samples were obtained from the birds 10-15 min after slaughter. The tissue samples were then immersion-fixed in Bouin's fluid for $12 \mathrm{~h}$. After fixation, tissues were processed routinely for histology and embedded in paraffin wax. The immunostaining technique was performed on $5 \mathrm{~lm}$ thick sections using a LSAB-plus kit (Dakocytomation, Denmark). Sections were deparaffinized and endogenous peroxidase activity was blocked, using a $3 \%(\mathrm{v} / \mathrm{v})$ hydrogen peroxide solution in water for $5 \mathrm{~min}$. The slides were then rinsed in a $0.01 \mathrm{~m}$ phosphate buffered saline solution (PBS, pH 7.4) for $5 \mathrm{~min}$. Thereafter, the slides were microwaved at 750 $\mathrm{W}$ for three cycles of $5 \mathrm{~min}$ each. After being allowed to cool for $20 \mathrm{~min}$ the sections were rinsed with PBS. The sections were then incubated for $30 \mathrm{~min}$ at room temperature with monoclonal antibodies against desmin, vimentin and SMA at dilutions of 1:300, 1:100 and 1:50 respectively. The antibodies were purchased from Dakocytomation, Denmark. After the incubation with primary antibodies the slides were rinsed with PBS and then incubated for 15 min with a biotinylated secondary antibody (LSAB-plus kit). Thereafter, the slides were rinsed in PBS and subsequently incubated for $15 \mathrm{~min}$ with the streptavidin peroxidase component of the LSAB-plus staining kit. Slides were then rinsed in PBS and bound antibody was visualized after the addition of a 3,34diaminobenzidine tetrachloride solution (LSAB-plus kit).

In the negative controls the primary antibodies were replaced with normal mouse serum. Smooth muscle was used as a positive control for both desmin and SMA, while tonsillar tissue was used as a positive control for vimentin. Variations in the immunostaining of sections used in this study were minor. No background staining was detected in the negative controls sections, while positive immunostaining for desmin, vimentin and SMA was observed in the control sections. Additional sections of emu ovarian tissue were stained with haematoxylin and eosin to provide a light microscopic description of the ovary. 


\section{Results}

\section{General overview}

The ovary was composed of a cortex, medulla and ovarian stalk. The cortex contained follicles at various stages of development, while the medulla and ovarian stalk contained large nerve bundles and blood vessels (Fig. 1a). Deep surface crypts partially divided the cortical region of the ovary into compartments. Lining the ovary was a simple cuboidal or columnar epithelium, which formed villous-like projections in some areas. The surface epithelium demonstrated vimentin immunoreactivity (Fig. 1b). Underlying the surface epithelium was a tunica albuginea, which was immunonegative for SMA and vimentin. Desmin immunopositive pericytes and smooth muscle cells were demonstrated in the tunica albuginea. An interesting feature of the emu ovary was the concentration of melanocytes at the interface of the tunica albuginea and the underlying cortical stroma (Fig. 1a). In some birds, the melanocytes extended into the cortical stroma. The cortical stroma was composed of cords of connective tissue separated by blood vessels with vimentin immunoreactive endothelial cells (Fig. 1b). The tunica media of the blood vessels displayed desmin and SMA immunoreactivity (Fig. 1c). Several lymphoid nodules of various sizes were observed mainly in the cortex. These nodules tended to occur close to developing follicles.

The connective tissue forming the medulla enclosed large bundles of smooth muscle, nerves and blood vessels. SMA andvdesmin immunoreactivity was particularly strong in the tunicavmedia of the blood vessels (Fig. 1d), as well as in thevperineurium of the nerve bundles. Strong vimentin immunoreactivityvwas limited to the endothelium of blood vessels. The medulla blended imperceptibly with the ovarian stalk. The components of the medulla were identical to those of the ovarian stalk.

\section{Developing follicles}

Primordial, pre-vitellogenic and vitellogenic follicles were identified in the ovary. Single primordial follicles were distributed in the cortex close to the surface epithelium. The primordial follicles (50$120 \mathrm{Im}$ in diameter) were composed of an oocyte enclosed by cuboidal granulosa cells. The granulosa cells were strongly immunopositive for vimentin (Fig. 1b), but immunonegative for desmin and SMA. Surrounding the granulosa cells was a layer of melanocytes and fibroblasts.

The smaller pre-vitellogenic follicles (120-500 $\mathrm{lm}$ in diameter) were situated close to the surface epithelium, with an indentation of the ovarian surface marking the location of these follicles. Larger pre-vitellogenic follicles (500-800 $\mathrm{lm}$ in diameter) were situated in the deeper layers of the cortex. The granulosa cell layer was composed of a simple cuboidal epithelium, which exhibited strong vimentin immunostaining. Surrounding the granulosa cell layer was a thecal layer composed of fibroblasts. 

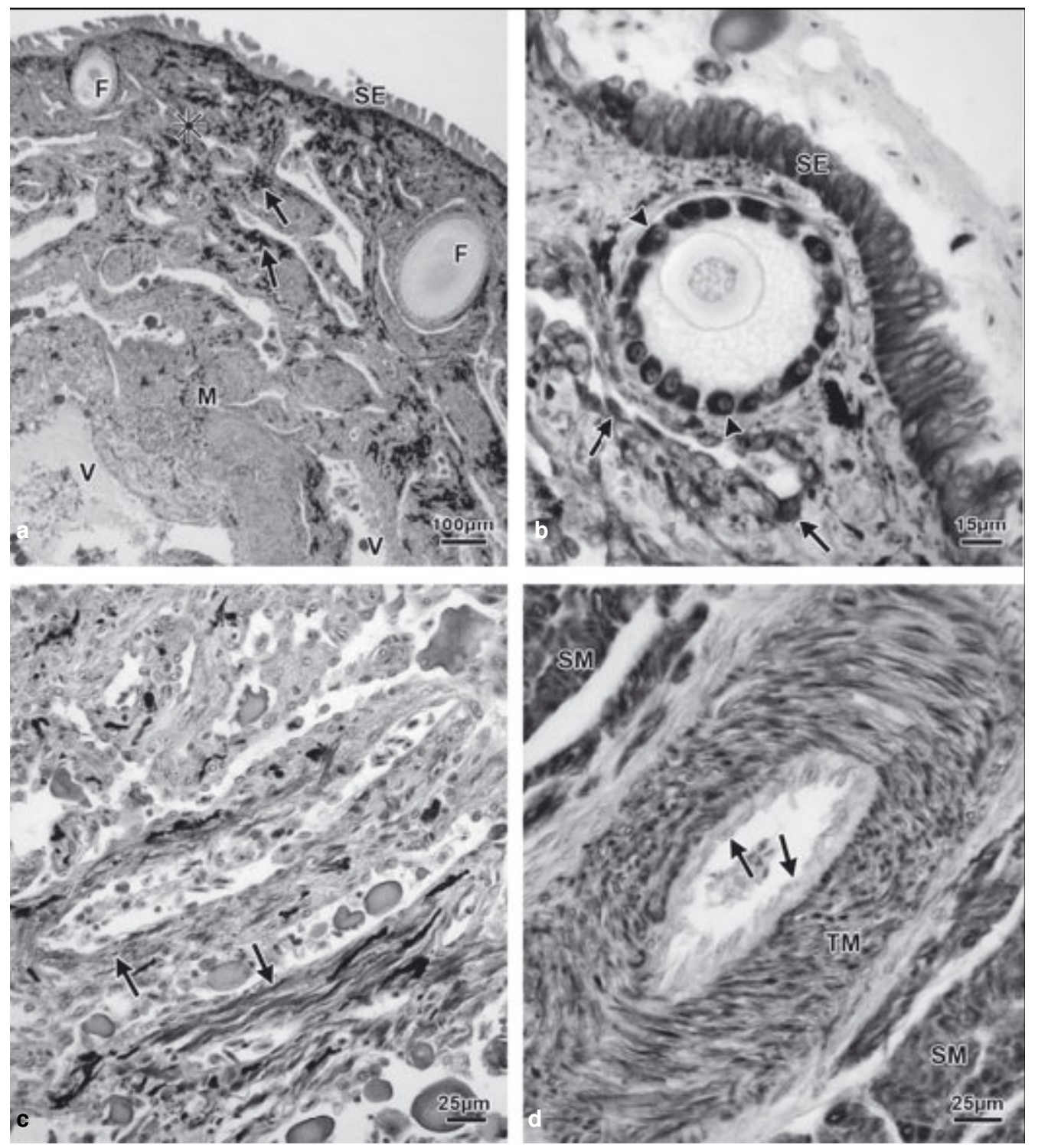

Fig. 1. (a) The cortical region (asterisk) of the ovary contains follicles $(F)$, while the medulla $(M)$ is characterized by the presence of large blood vessels (V). Note the presence of numerous melanocytes (arrows) in the cortical cords. Surface epithelium (SE), haematoxylin and eosin. (b) Vimentin immunoreactivity in the surface epithelium (SE), endothelial cells (arrows), as well as in the granulosa cell layer (arrowheads) of a primordial follicle. (c) Smooth muscle actin (SMA) immunoreactive tunica media (arrows) of blood vessels situated in the cortical cords. (d) Strong desmin immunoreactivity is demonstrated in the tunica media (TM) of a blood vessel, as well as in the adjacent smooth muscle bundles (SM) in the medulla. The endothelial lining (arrows) of the blood vessel is immunonegative for desmin.

Early vitellogenic follicles (800-2300 Im in diameter) were characterized by an oocyte partially filled with yolk. The granulosa cell layer was simple to pseudostratified columnar. Moderate vimentin immunoreactivity was observed in the basal regions of the granulosa cells (Fig. 2a). The thecal layer was clearly differentiated into theca externa and theca interna. The theca interna close to the granulosa cell layer contained a terminal capillary network, which was enclosed by a layer of desmin immunoreactive pericytes (Fig. 2b). Surrounding the theca externa was the superficial tunic, which contained connective tissue, intramural smooth muscle bundles and 
external intramural blood vessels. The endothelial lining of the blood vessels was vimentin immunoreactive (Fig. 2c). Strong desmin andSMAimmunoreactivity (Fig. 2d) was demonstrated in the intramural muscle bundles.

In late vitellogenic follicles ( $\$ 2300 \mathrm{Im}$ in diameter) the granulosa cell layer was simple cuboidal. The granulosa cells were immunonegative for desmin (Fig. 3a) and SMA (Fig. 3b), but immunopositive for vimentin (Fig. 3c). Underlying the granulosa cell layer was a theca interna composed of fibroblasts. The endothelial lining of the terminal capillary network within the theca interna showed strong vimentin immunostaining (Fig. 3c). Strong SMA immunoreactivity was observed in the fibroblasts forming the theca externa (Fig. 3b). Traversing the theca externa were branches of the intramural artery and middle intramural vein. The endothelial lining of these blood vessels expressed vimentin immunoreactivity.

SMA immunoreactivity was demonstrated in the tunica media of the branches of the intramural artery. The superficial tunic enclosing the follicle contained intramural muscle bundles, which were strongly immunopositive for desmin and SMA (Fig. 3b).

\section{Atretic follicles}

Atresia in primordial and pre-vitellogenic follicles was marked by the presence of a shrunken oocyte surrounded by folds of a vimentin immunoreactive granulosa cell layer. In the advanced stages of atresia, the primordial and pre-vitellogenic follicles were infiltrated by stroma. Ultimately, the site of follicular atresia was discernible only by a dense accumulation of melanocytes.

The early stages of atresia in vitellogenic follicles were marked by the proliferation of the granulosa and theca interna cells. The proliferating granulosa cells were immunopositive for vimentin, while the theca interna cells did not display vimentin immunoreactivity. Capillary networks were evident in the theca interna and theca externa. The endothelial cells lining the capillaries were vimentin immunopositive (Fig. 4a), while the surrounding pericytes were desmin immunoreactive (Fig. 4b). The endothelial cells and pericytes were SMA immunonegative (Fig. 4c). At this stage the middle and external intramural veins located at the outer limits of the theca externa and in the superficial tunic respectively, underwent a major transformation, with the endothelial cells becoming columnar in shape. Vimentin immunoreactivity was demonstrated in the modified endothelial cells (Fig. 4a).

The granulosa and theca interna cells subsequently differentiated into interstitial gland cells, which infiltrated both the theca externa and oocyte. In some birds, melanocytes were distributed between the gland cells (Fig. 5a). Eventually, the interstitial gland cells abutted the modified 
intramural veins (Fig. 5a). Extending between die gland cells were strands of connective tissue and capillaries.
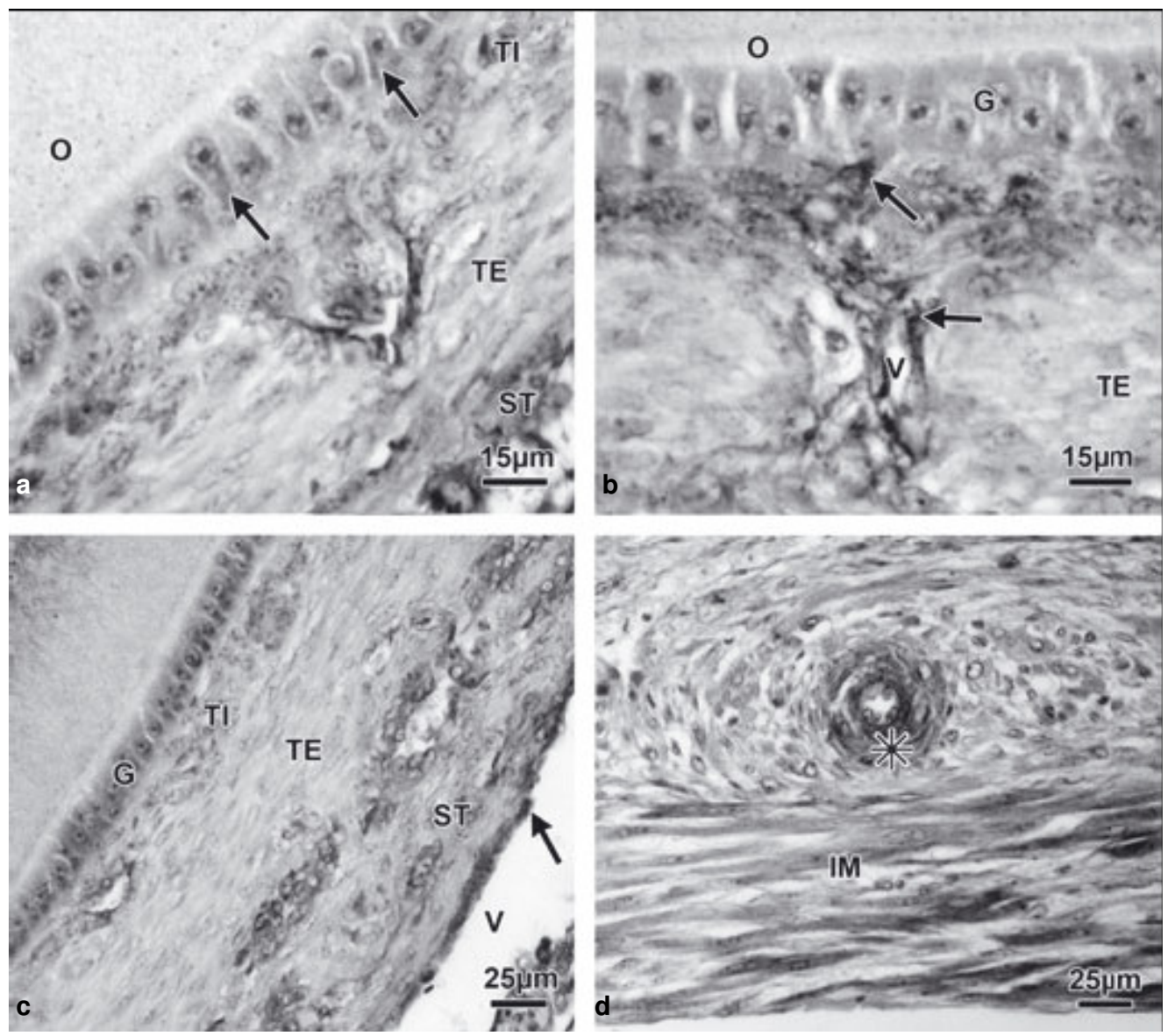

Fig. 2. Early vitellogenic follicle. (a) Vimentin immunoreactivity occurs in the basal regions (arrows) of the pseudostratified columnar granulosa cell layer. O, oocyte; TE, theca externa; TI, theca interna; ST, superficial tunic. (b) Desmin immunoreactive pericytes (arrows) enclose capillaries in the theca interna. G, granulosa cell layer; O, oocyte; V, a vascular branch traversing the theca externa (TE). (c) The superficial tunic (ST) contains external intramural veins (V), which are lined by vimentin immunopositive endothelial cells (arrow). G, granulosa cell layer; TE, theca externa; TI, theca interna. (d) Smooth muscle actin (SMA) immunoreactivity is exhibited in the tunica media (asterisk) of an intramural artery, as well as in an intramural muscle bundle (IM) of the superficial tunic. 

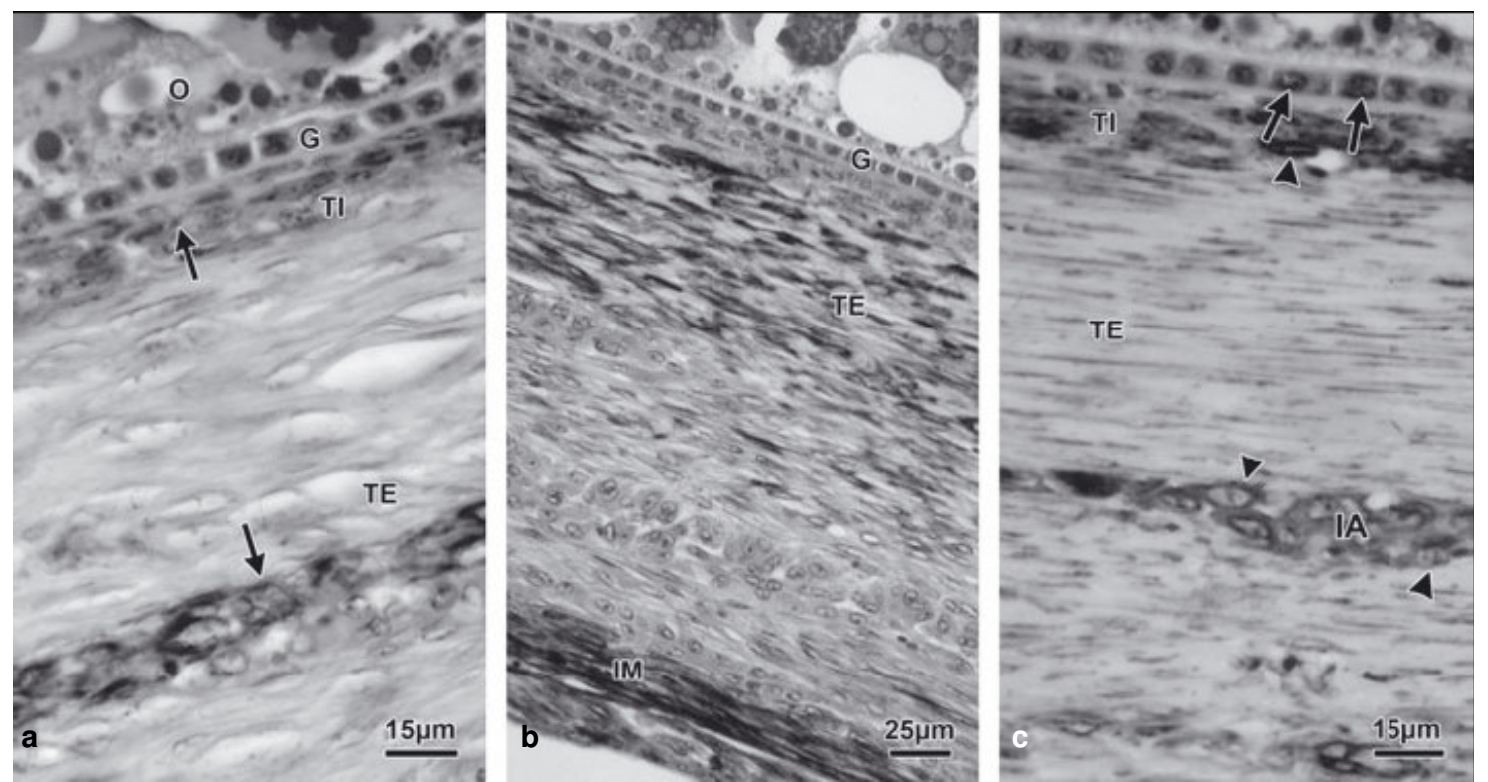

Fig. 3. Late vitellogenic follicle. (a) The granulosa cell layer $(G)$ is desmin immunonegative. Desmin immunoreactivity is displayed in pericytes (arrows) of the terminal capillary network and in smooth muscle cells of an intramural blood vessel. $\mathrm{O}$, oocyte; TE, theca externa; TI, theca interna. (b) Strong smooth muscle actin (SMA) immunoreactivity is demonstrated in the theca externa (TE), as well as in intramural muscle bundles (IM) located in the superficial tunic. G, SMA immunonegative granulosa cell layer. (c) Granulosa cells (arrows) contain a thin rim of vimentin immunoreactive cytoplasm. Vimentin immunoreactivity is also exhibited by endothelial cells (arrowheads) of the terminal capillary network and the intramural artery (IA). TE, theca externa; TI, theca interna.
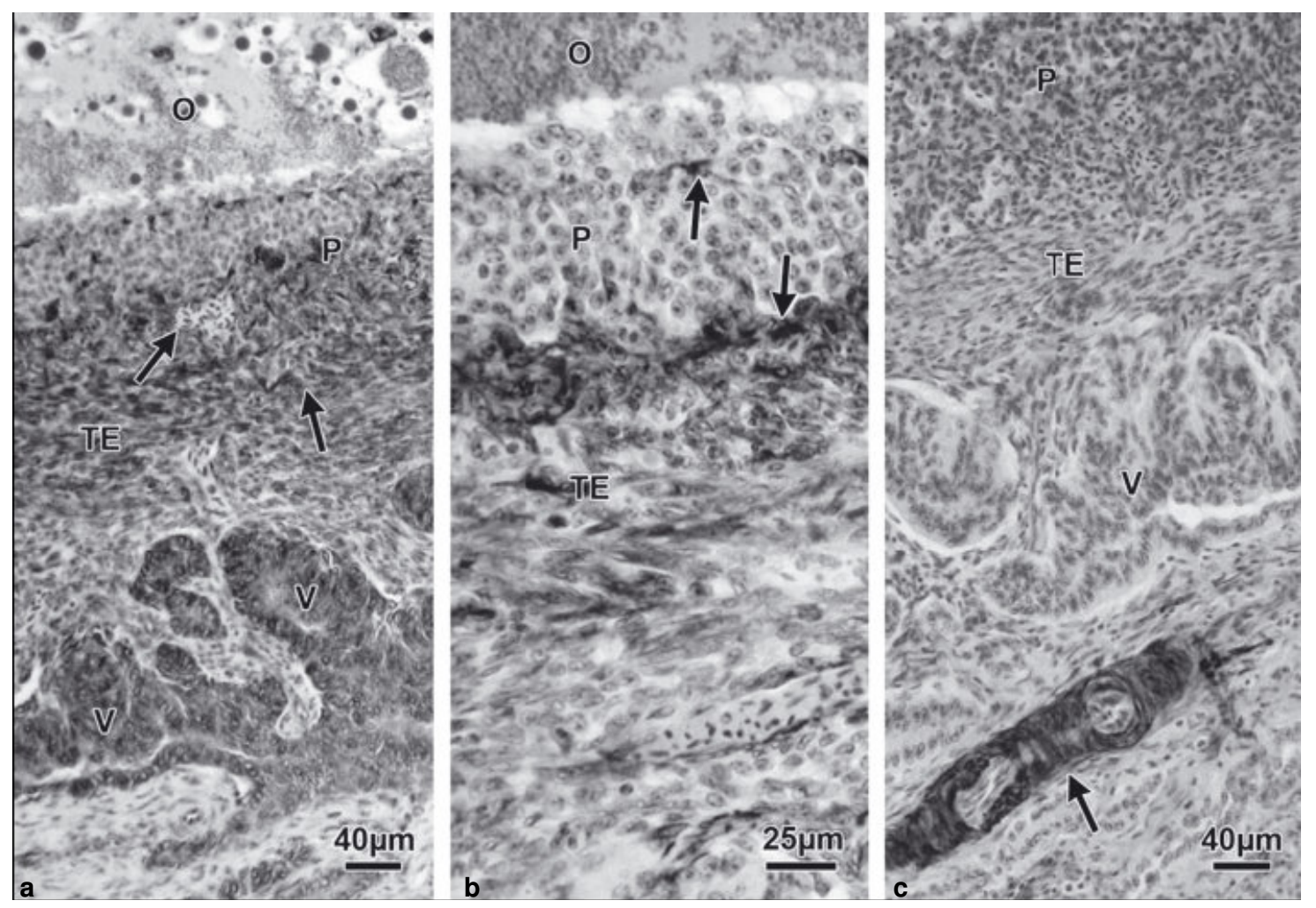

Fig. 4. Atretic vitellogenic follicle. (a) Vimentin immunoreactivity is demonstrated in the endothelial cells (arrows) of capillaries in the theca externa (TE) and proliferating layer ( $P$ ), as well as in the endothelial lining of middle intramural veins $(\mathrm{V})$. O, oocyte. (b) Pericytes (arrows) surrounding capillaries in the theca externa (TE) and proliferating layer $(\mathrm{P})$ display desmin immunoreactivity. O, oocyte. (c) The capillary network in the theca externa (TE) and proliferating layer (P) 
is immunonegative for smooth muscle actin (SMA). SMA immunoreactivity is present in the tunica media (arrow) of a blood vessel in the superficial tunic. $V$, middle intramural vein.
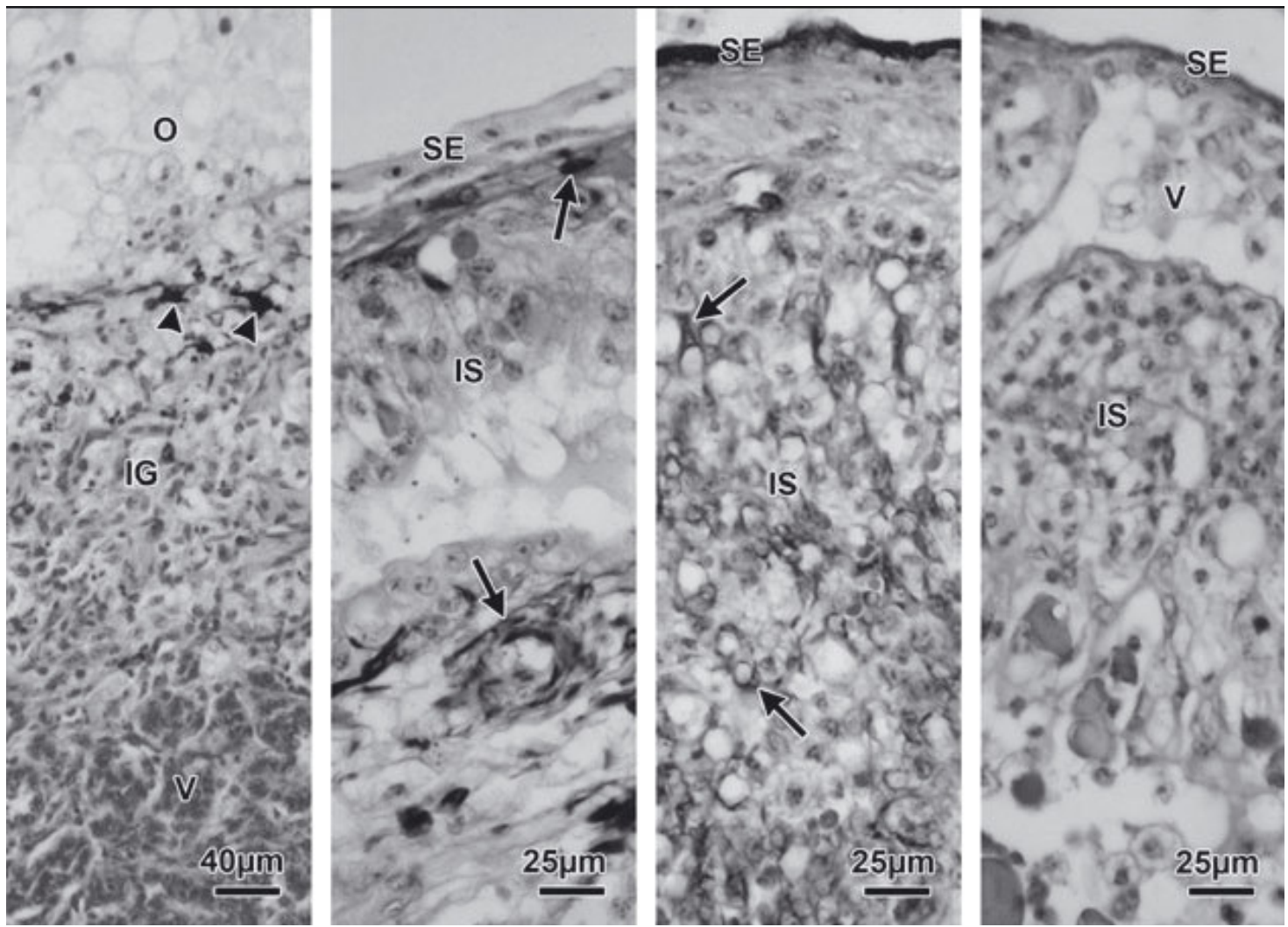

Fig. 5. Interstitial gland cells in an atretic vitellogenic follicle (a) and in the cortical stroma (b-d). (a) Interstitial gland cells (IG) have infiltrated the theca externa and are in close proximity to the middle intramural veins (V). Strands of connective tissue and melanocytes (arrowheads) are interspersed between the gland cells. O, oocyte; haematoxylin and eosin. (b). Desmin immunoreactivity (arrows) is present in pericytes and smooth muscle cells situated between interstitial stromal gland cell masses (IS) and within the tunica albuginea. SE, surface epithelium. (c) Vimentin immunoreactivity is demonstrated in the surface epithelium (SE), as well as in some gland cells and endothelial cells (arrows). IS, interstitial stromal gland cells. (d) The interstitial stromal gland cells (IS) and associated blood vessels (V) are smooth muscle actin (SMA) immunonegative. SE, surface epithelium.

The interstitial gland cells eventually dispersed to form stromal interstitial gland cells, which amassed around the intramural veins. The stromal interstitial gland cells and associated blood vessels were typically situated in the upper limits of the cortex. The stromal interstitial gland cells were immunonegative for desmin (Fig. 5b) and SMA (Fig. 5d). A combination of vimentin immunopositive and immunonegative stromal interstitial gland cells was evident (Fig. 5c). In addition, capillaries with vimentin immunoreactive endothelial cells were observed between the interstitial gland cells.

\section{Discussion}

The present study was undertaken to provide a comparison of the distribution of intermediate filaments in the ovary of the emu with that of other birds, as well as with mammals. Although the present study highlighted many similarities in the occurrence of intermediate filaments in the emu 
compared with the Japanese quail (Van Nassauw et al., 1989, 1992; Van Nassauw and Callebaut, 1991) and ostrich (Madekurozwa and Kimaro, 2006a), differences were also noted.

Immunohistochemical studies conducted on the ovaries of both birds (Madekurozwa and Kimaro, 2006a) and mammals (Czernobilsky et al., 1985; Khan-Dawood et al., 1996; Marettova and Maretta, 2002) have localized the intermediate filament vimentin in granulosa cells, endothelial cells, as well as in the surface epithelium of the ovary. The occurrence of vimentin immunoreactivity in these regions of the ovary is variable, with immunoreactivity to vimentin demonstrated in the ovarian surface epithelium of the emu, ostrich (Madekurozwa and Kimaro, 2006a) and primates (Czernobilsky et al., 1985; Khan-Dawood et al., 1996), but not in the pig (Czernobilsky et al., 1985) and bovine (Van den Hurk et al., 1995). Likewise, although vimentin immunostaining is generally present in granulosa cells, it is absent in the granulosa cell layer of primates (Khan-Dawood et al., 1996). In both the emu and the ostrich (Madekurozwa and Kimaro, 2006a), vimentin immunoreactivity was strong in the granulosa cells of developing and atretic primordial and pre-vitellogenic follicles. However, vimentin immunoreactivity was present in the granulosa cell layer of developing and atretic vitellogenic follicles in the emu, but was absent in the granulosa cell layer of vitellogenic follicles in the ostrich (Madekurozwa and Kimaro, 2006a). A noteworthy feature of the current study was the use of vimentin immunoreactivity to distinguish between proliferating and interstitial gland cells derived from granulosa and theca interna cells. Vimentin immunoreactivity clearly showed that both the proliferating and interstitial layer are composed of both granulosa and theca interna cells.

The intermediate filament desmin is localized in all muscle types, while SMA is specific for smooth muscle (Skalli et al., 1986). In the ovary of the emu, strong desmin and SMA immunoreactivity was demonstrated in the tunica media of blood vessels in the cortex and medulla. In addition, desmin and SMA immunostaining was displayed in the tunica media of blood vessels in the connective tissue layers of developing and atretic vitellogenic follicles. These results correlate well with findings in the turtle (Van Nassauw et al., 1991), Japanese quail (Van Nassauw and Callebaut, 1991; Van Nassauw et al., 1992), rat (Selstam et al., 1993), sheep (Marettova and Maretta, 2002) and bovine (Van den Hurk et al., 1995). The presence of desmin and SMA in the tunica media of blood vessels suggests that these intermediate filaments play a role in the regulation of blood flow through the ovary. In the emu ovary, desmin and SMA were demonstrated not only in the tunica media, but also in intramural muscle bundles situated in the superficial tunic. Similar findings have been reported in the Japanese quail (Van Nassauw and Callebaut, 1991; Van Nassauw et al., 1992) and the turtle (Van Nassauw et al., 1991). It is thought that the intramural muscle bundles, in conjunction with smooth muscle in the tunica albuginea, play a role in the formation of a structural framework, which provides mechanical 
support to the ovary (Van Nassauw et al., 1989). In addition, the intramural muscle bundles are thought to be involved in the rupture of the follicular wall during ovulation (Van Nassauw et al., 1989). Likewise, SMA immunopositive cells in the theca externa are also thought to be involved in the ovulatory process (Martin and Talbot, 1981; Van Nassauw et al., 1993). Furthermore, research conducted on atretic follicles in the mouse suggests that theca externa cells are involved in the infolding of the follicular wall during atresia (Motta and Familiari, 1981). In addition to being demonstrated in the emu, SMA immunoreactive theca externa cells have also been identified in the Japanese quail (Van Nassauw and Callebaut, 1991; Van Nassauw et al., 1992), ostrich (Madekurozwa and Kimaro, 2006a) and sheep (Marettova and Maretta, 2002). Although theca externa cells express SMA immunoreactivity, ultrastructural studies in the domestic fowl (Rothwell and Solomon, 1977; Perry et al., 1978) and ostrich (Madekurozwa and Kimaro, 2006b) indicate that these cells are fibroblasts, not smooth muscle cells. However, based on the presence of SMA immunoreactivity in the theca externa, it is clear that these fibroblasts possess certain characteristics of smooth muscle cells. Indeed, research has shown that fibroblasts in the theca externa of the domestic fowl (Yoshimura et al., 1983) and the Japanese quail (Van Nassauw et al., 1993) possess a contractile ability.

In the ostrich, SMA and desmin immunoreactive fibroblastlike cells were prominent features of atretic vitellogenic follicles (Madekurozwa and Kimaro, 2006a). Although the stages of atresia in the emu appear similar to those of the ostrich, the SMA and desmin immunoreactive cells were not observed in the emu. Instead, atretic follicles in the emu were characterized by the presence of numerous melanocytes, the function and significance of which is unclear.

In conclusion, the results of the present study indicate that the distribution of intermediate filaments in the ovary of the emu is similar in many respects to that in the ostrich ovary (Madekurozwa and Kimaro, 2006a). However, differences were noted in the atretic vitellogenic follicles with regards to the absent of desmin and SMA immunoreactive fibroblast-like cells, which were prominent in the ostrich (Madekurozwa and Kimaro, 2006a).

\section{Acknowledgements}

The author thanks staff in the Department of Pathology, Department of Physiology and the Department of Education Innovation (Creative Studios) for their assistance. The University of Pretoria and the National Research Foundation funded this study. 


\section{References}

Czernobilsky, B., R. Moll, R. Levy, and W. W. Franke, 1985: Co-expression of cytokeratin and vimentin filaments in mesothelial, granulosa and rete ovarii cells of the human ovary. Eur. J. Cell Biol. 37, 175-190.

Galou, M., J. Gao, J. Humbert, M. Mericskay, Z. Li, D. Paulin, and P. Vicart, 1997: The importance of intermediate filaments in the adaptation of tissues to mechanical stress: evidence from gene knock out studies. Biol. Cell. 89, 85-97.

Goldman, R. D., S. Khuon, Y. H. Chou, P. Opal, and P. M. Steinert, 1996: The function of intermediate filaments in cell shape and cytoskeletal integrity. J. Cell. Biol. 134, 971-983.

Khan-Dawood, F. S., M. Y. Dawood, and S. Tabibzadeh, 1996: Immunohistochemical analysis of the microanatomy of primate ovary. Biol. Reprod. 54, 734-742.

Madekurozwa, M.-C., and W. H. Kimaro, 2006a: A morphological and immunohistochemical study of healthy and atretic follicles in the ovary of the sexually immature ostrich (Struthio camelus). Anat. Histol. Embryol. 35, 253-258.

Madekurozwa, M.-C., and W. H. Kimaro, 2006b: Ultrastructural features of the follicular wall in developing follicles of the sexually immature ostrich (Struthio camelus). Onder. J. Vet. Res. 73, $199-205$.

Marettova, E., and M. Maretta, 2002: Demonstration of intermediate filaments in sheep ovary. Acta Histochem. 104, 431-434.

Martin, G. G., and P. Talbot, 1981: The role of follicular smooth muscle cells in hamster ovulation. J. Exp. Zool. 216, 469-482.

Minnaar, P., and M. Minnaar, 1997: The breeding season. In: The Emu Farmer's Handbook (P. Minnaar, ed.). Groveton, TX: Nyoni Publishing Co., pp. 75.

Motta, P. M., and G. Familiari, 1981: Occurrence of a contractile tissue in the theca externa of atretic follicles in the mouse ovary. Acta Anat. 109, 103-114.

Perry, M. M., A. B. Gilbert, and A. J. Evans, 1978: Electron microscope observations on the ovarian follicle of the domestic fowl during the rapid growth phase. J. Anat. 125, 481-497.

Rothwell, B., and S. E. Solomon, 1977: The ultrastructure of the follicle wall of the domestic fowl during the phase of rapid growth. Br. Poult. Sci. 18, 605-610.

Selstam, G., I. Nilsson, and M.-O. Mattsson, 1993: Changes in the ovarian intermediate filament desmin during the luteal phase of the adult pseudopregnant rat. Acta Physiol. Scand. 147, 123- 129.

Skalli, O., P. Ropraz, A. Trzeciak, G. Benzonana, D. Gillessen, and G. Gabbiani, 1986: A monoclonal antibody against alpha-smooth muscle actin: a new probe for smooth muscle differentiation. J. Cell. Biol. 103, 2787-2796. 
Van den Hurk, R., G. Dijkstra, F. N. Van Mil, S. C. J. Hulshof, and S. G. A. M. Van den Ingh, 1995: Distribution of the intermediate filament proteins vimentin, keratin and desmin in the bovine ovary. Mol. Reprod. Dev. 41, 459-467.

Van Nassauw, L., and M. Callebaut, 1991: Structural and immunohistochemical aspects of the postovulatory follicle in Japanese quail. Anat. Rec. 229, 27-30.

Van Nassauw, L., M. Callebaut, F. Harrisson, G. Daneels, and M. Moeremans, 1989: Immunohistochemical localization of desmin in the quail ovary, demonstration of a suspensory apparatus. Histochemistry 90, 371-377.

Van Nassauw, L., F. Harrisson, and M. Callebaut, 1991: Localization of smooth-muscle markers in the ovaries of some ectothermic vertebrates. Anat. Rec. 229, 439-446.

Van Nassauw, L., F. Harrisson, and M. Callebaut, 1992: Immunolocalization of smooth musclelike cells in the quail ovary. Eur. J. Morphol. 30, 275-288.

Van Nassauw, L., S. U. Sys, F. Harrisson, and M. Callebaut, 1993: In vitro study of the contractility of the wall of the preovulatory follicle in the Japanese quail. Biol. Reprod. 49, 359364.

Yoshimura, Y., K. Tanaka, and O. Koga, 1983: Studies on the contractility of follicular wall with special reference to the mechanism of ovulation in hens. Br. Poult. Sci. 24, 213-218. 